\title{
Longitude Distribution of
}

\section{Solar Flares}

by

P. C.W. Fung, P. A. Sturrock,

P. Switzer, G. Van Hoven

CSEPY

SU-IPR Report No.393

November 1970
Air Force Office of Scientific Research
Contract F44620-69-C-0008
and

National Aeronautics and Space Administration

Grant NGL 05-020-272

\author{
INSTITUTE FOR PLASMA RESEARCH \\ STANFORD UNIVERSITY, STANFORD, CALIFORNIA
}


LONGITUDE DISTRIBUTION OF SOLAR FLARES

by

P.C.W. Fung, P.A. Sturrock, P. Switzer, G. Van Hoven

\author{
Air Force Office of Scientific Research \\ Contract F44620-69-C-0008 \\ and \\ National Aeronautics and Space Administration \\ Grant NGL 05-020-272
}

SU-IPR Report No. 393

November 1970

This document has been approved for public release and sale; its distribution is unlimited.

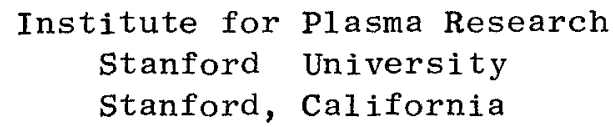


Qualified requesters may obtain additional copies from the Defense Documentation Center. All others should apply to the Clearing House for Federal Scientific and Technical Information. 


\title{
LONGITUDE DISTRIBUTION OF SOLAR FLARES
}

\author{
By \\ P.C.W. Fung ${ }^{*}$, P.A. Sturrock ${ }^{\dagger}$, P. Switzer ${ }^{* *}$, G. Van Hoven ${ }^{\dagger \dagger}$ \\ Stanford University, Stanford, California \\ Abstract
}

Statistical tests, based on the maximum-likelihood method, have been performed on flare series extending over several years. If all flares in each plage region are taken into account, a rich spectrum is abtained. If one carries out similar analysis of a "reduced" flare series, which includes at most one flare from each plage region, the spectrum is almost devoid of structure, and what structure does remain is not statistically significant. The inference is that solar activity does not display genuine rigid-rotation modulation, but that repeated events from individual centers of activity yield modulation which may be mistaken for rigid-rotation modulation.

A test for correlation between reduced flare series for the northern and southern hemispheres gives no significant correlation. This test therefore yields no support to the hypothesis that solar activity is modulated by planetary effects.

\footnotetext{
* Present address: Department of Physics, University of Hong Kong

†Istitute for Plasma Research and Department of Applied Physics

** Department of statistics

${ }^{+}$Present address: Department of Physics, University of California at Irvine
} 


\section{Introduction}

It was shown by Carrington (1863) that, if sunspots are taken as markers, the visible surface of the sun exhibits differential rotation. The synodic rotation period (that is, the period as viewed from the earth) is about 26.9 days at the solar equator and 33.9 days at the solar poles (Newton and Nunn, 1951). The "Carrington period" (27.275 days) is the conventional average period used for reporting solar longitude data (Explanatary Supplement to the Astronomical Ephemeris and the American Ephemeris and Nautical Almanac, 1961). In view of this differential rotation, one would not expect any visible or detectable solar phenomenon to exhibit a strict long-term periodicity. Nevertheless, there has for some time been indications that long term periodicity does exist.

It has been known for some time that geomagnetic storms of moderate. intensity often recur at intervals of about 27 days. Bartels (1932) called the solar source of recurring geomagnetic storms an "M region". Important information concerning this phenomenon was obtained by Wilcox and Ness (1965) who found that the interplanetary magnetic field displays a "sector structure" which survives many solar rotations. The mean rotation period of this structure is about 27 days. More recently, Wilcox, Schatten, Tannenbaum and Howard (1970) have shown that an autocorrelation function formed from the photospheric magnetic field exhibits some behavior characteristic of differential rotation and some behavior characteristic of rigid rotation with a period of about 27 days. It seems, therefore, that there is at this time strong evidence. that weak-field phenomena exhibit rigid-body rotation, although the cause of this effect is not known. 
There has been much discussion in the literature of possible rigidbody rotation associated with what we might call "strong-field phenomena": namely, sunspots and solar flares. Sawyer-Warwick (1965) reports earlier work on sunspot distributions by Losh (1938) and Vitinskii (1960), and shows that both studies exhibit significant structure when referred to the Carrington period. Sawyer-Warwick points out that the distributions found by Losh for solar cycles 14 through 17 peak at approximately the same longitudes. However, the distributions found by Vitinskii for solar cycles 12 through 18 show little evidence for consistency of longitudes of peak activity. A further drawback in the appraisal of the evidence concerning sunspots is that longitude distributions are typically given only for one choice of rotation rate of the longitude coordinate system.

Guss (1964) and Sawyer-Warwick (1965) have both presented evidence that proton-producing solar flares occur preferentially in certain longitude intervals. Guss considered 56 such events occurring between 16 January 1955 and 23 October 1962, When referred to a coordinate system with synodic rotation rate 27.04 days, the distribution of flares appears to be significantly nonuniform. Sawyer-Warwick considered flares producing polar-cap absorption (PCA) events in the period 1954 to 1958. There were 78 such events, but Sawyer-Warwick compiled a "reduced series" with the property that only one event occurred in any one active region: the reduced flare series comprised 45 events. When referred to the longitude system associated with the Carrington rotation period, this distribution also appears to be significantly nonuniform.

Wilcox and Schatten (1967) pointed out that there is no a priori reason to consider the Carrington rotation period (or that adopted by Guss) 
rather than any other period comparable with the range characterizing solar differential rotation. Working with the reduced flare series compiled by Sawyer-Warwick, they investigated the nonuniformity as a function of synodic rotation period over the range 25 days to 34 days. The dependence on rotation period of the degree of non-uniformity (measured by the number of flares in the least-populated hemisphere) was found to be very similar to that of 45 events distributed at random over the same time interval. Hence, when tested over a wide range of rotation periods, there appeared to be no evidence for significant nonuniformity of the longitude-distribution of the reduced flare series compiled by Sawyer-Warwick.

Although the analysis of Wilcox and Schatten argues against the existence of longitude-grouping of solar flares, it seemed that it would be worthwhile to carry out a more extensive investigation. This . view was motivated partly by the strong evidence that weak-field phenomena display rigid-rotation behavior, and partly by the prospect that rigidbody rotation, if it exists, might be attributed to the radiative core and/or the convective zone. The proposal by Dicke and Goldenberg (1967) that the sun's core rotates with a period of about two days led to renewed interest in study of the sun's rotation. It seemed possible that nonuniformity of a rigidly rotating core might trigger flares in such a way that flares would be found to cluster in longitude when referred to a coordinate system with the rotation rate of the core. Although a similar effect might possibly exist for sunspots, the long lifetimes of sunspots make them unsuitable for study of longitude-distributions associated with short rotation periods.

Since our first aim was to test for longitude-clustering which might be associated with real rigid-body rotation, it seemed unnecessary 
to work with a reduced flare series. A series of 1119 flares of importance greater than or equal to $2-$, distributed over $6 \frac{1}{2}$ years of solar cycle 19, was spectrum-analyzed in longitude by a statistical procedure (Sections 2 and 3 ) incorporating the maximum-likelihood technique (Rao, 1965). A rich spectrum was found but, for reasons to be given later (Section 4), it was decided that the spectrum gave no firm evidence of rigid-body rotation.

In order to test whether the above results were to be attributed to long-lived centers of activity, a reduced flare series was compiled, comprising 2,670 "independent" flares occurring between January 1,1956, and December 31, 1968. Analysis of this flare series will be presented in Section 5. Results of the analyses of Sections 4 and 5 will be discussed in Section 6 .

2. Maximum Likelihood Estimation of Flare-Series Spectrum

In order to search for the possibility that rigid-body rotation underlies flare occurrences, it is necessary to consider different hypothetical rotation rates and find longitude distributions for each rate. The raw data, therefore will be a distribution of flares in time and longitude (referred to some conventional rotating frame). We assume that the flare occurrence may be adequately modeled by a stochastic event-process for which the probability of the occurrence of the flare is a function of time and longitude only (i.e, it is a Poisson point process [Cox and Lewis, 1966] with a varying intensity function). This modeling permits us to use the maximum-1ikelihood method (Rao, 1965) to estimate unknown parameter values of the intensity function.

In its simplest form, the method of maximum-likelihood involves a testing of a postulated intensity function $f(\theta ; \beta)$ for events occurring 
at "time" $\theta_{2}$ the form of the function $f$ depending on the parameter (or parameters) $\%$. The relative probability that the data set $\left(\theta_{1}, \ldots, \theta_{N}\right)$ may be attributed to various values of $\beta$ is estimated from the likelihood function

$$
L\left(\theta_{1}, \ldots, \theta_{N} ; \beta\right)=\prod_{n=1}^{N} f\left(\theta_{n} ; \beta\right)
$$

The value of the parameter $\beta$ which is "most likely" to make the model consistent with the data is that which maximizes the likelihood. Provided certain conditions concerning regularity and smoothness are satisfied (Rao, 1965), maximum-1ikelihood estimators have two important properties. They are "consistent" estimators of the parameters in the sense that the probability that the estimated value of a parameter will differ from the true value by any specified amount becomes vanishingly small as the sample size increases. Second, the asymptotic (asymptotic with respect to the sample size, N) sampling distribution of estimated values is normal with variances which are not greater than those for any other estimation method.

\section{A Model for the Solar-Flare Distribution}

For present purposes, a flare will be characterized by its longitude $\ell$ and time $t$ of occurrence; latitude need not be considered since we are searching for rigid-body rotation. Observational records assign to a flare a "central meridian distance" $\lambda$. This corresponds to a measurement of solar longitude referred to the "subterrestrial point" as origin. This longitude system is therefore tied to the earth's orbital motion, and in the following we treat this motion as being characterized by a 
constant rotation rate $\omega_{E}$. (The fact that the earth's.orbital motion is not strictly uniform involves certain annually varying corrections $\Delta \lambda$ to $\lambda$ which will not be specified but which have been taken into account.)

When referred to a frame rotating with arbitrary sidereal angular velocity $\omega_{I}$, the running longitude $\ell$ of a flare will be given by

$$
\ell=\lambda+\left(\omega_{I}-\omega_{E}\right) t
$$

where $\lambda$ is the corrected value and we adopt the convention that $\ell=\lambda$ at $t=0$. Equation (2) may be rewritten as

$$
\ell=\lambda+\omega_{Y} t
$$

where $\omega_{\mathrm{Y}}$ is the synodic rotation rate.

The following simple form for the test function was adopted:

$1+\delta \sin (\ell-\gamma)$. However, it was convenient to use in place of $t$ the variable $\theta$, where

$$
\theta=w_{C} t
$$

$\omega_{C}$ being the Carrington rotation rate. The trial function was therefore written as

$$
f(\theta, \lambda ; \alpha, \delta, \gamma)=A^{-1}[1+\delta \sin (\alpha \theta+\lambda-\gamma)]
$$




$$
\alpha=\omega_{Y} / \omega_{C}
$$

and $A$ is a normalization parameter depending on $\alpha, \delta, \gamma$ and the end points $\theta_{A}, \theta_{B}$ of the data range.

This model satisfies the regularity conditions necessary for consistency of maximum-1ikelihood parameter estimates and consequently the estimates obtained are asymptotically unbiased, efficient, and normally distributed with calculable variances. This model leads to the following approximate analytic expressions for the variances (derived from the corresponding diagonal elements of the inverse information matrix):

$$
\left.\begin{array}{l}
\sigma_{\alpha}^{2} \approx 24 N^{-1}\left(\theta_{B}-\theta_{A}\right)^{-2} \delta^{-2}\left(1-\frac{1}{4} \delta^{2}\right), \\
\sigma_{\delta}^{2} \approx 2 N^{-1}\left(1-\frac{3}{4} \delta^{2}\right), \\
\sigma_{Y}^{2} \approx 8 N^{-1}\left(\theta_{B}^{3}-\theta_{A}^{3}\right)\left(\theta_{B}-\theta_{A}\right)^{-3} \delta^{-2}\left(1-\frac{1}{4} \delta^{2}\right) .
\end{array}\right\}
$$

Because likelihood estimates are distributed normally in the asymptotic limit, we can answer the question "With what probability can the structure found in this model be explained as a manifestation of a purely random flare distribution?" by determining from the estimates of $\delta$ and its standard deviation the significance level of the hypothesis that $\delta=0$.

\section{Statistical Analysis of the Flare Series}

The above estimation methods have been applied to a large sample of solar flares and also to artificially generated random series. The 
flare sample was taken from the world-wide list compiled and statistically normalized by Sawyer-Warwick (1962, 1964, 1966)。This compilation covers the period July 1, 1955, to December 31, 1961, of solar cycle 19 but we have selected from this compilation those flares with importance $\geq 2-$. If the commencement of a flare was not observed by any station, it has been eliminated. This selection yielded a sample size of $N=1119$, distributed over the maximum of solar activity. The estimation procedure has been applied to artificially generated uniform random series and to artificially generated sinusoidal distributions simulating flare samples with nonzero modulation. This testing with specifically distributed flare series has been used to explore the limits of the sinusoidal model. The only weakness that we have found is an insensitivity to weak modulation (small values of $\delta$ ). This insensitivity originates in a subsidiary calculation necessary to start the iteration process. This calculation, similar to that used in periodogram analysis (Jenkins 1967), provides an estimate of $\delta^{2}$. Since this corresponds to a positive initial value for $\delta$, the maximumlikelihood iteration invariably finds $|\delta|$. This estimate of $\delta$ may be misleading in that $|\delta|$ necessarily has a nonzero expectation, even when the true value of $\delta$ is zero. However, this effect (which is similar to a bias which diminishes as $\delta$ increases) is unimportant if $\delta>0.2$.

Trials with artificially generated flare distributions, for which the depths of modulation were equal to those found for the real sample, have demonstrated that the essential estimates approach consistency in a satisfactory asymptotic manner. The values of $\alpha, \delta$ and $\gamma$ can be estimated satisfactorily from a sample of 1000 events as shown by the 
t-test for bias. There are discrepancies in the standard deviations of $\alpha$ and $\gamma$ as shown by the $x^{2}$-test for variance ratio. The largest values of these discrepancies were obtained when a long-term (11 years) modulation was added to the artificial series; when this additional variation had a depth of modulation similar to that which in fact occurs as a result of the solar cycle, the standard deviation of $\alpha$ was underestimated by 40 percent and that of $\gamma$ by 50 percent. A small bias and underestimation of the variance in $\delta$, which existed when the long-term variation was not considered, disappeared when the solar-cycle modulation was included.

These tests with artificially generated series provide information concerning the only significant problem involved in using the maximumlikelihood method, namely the effectiveness of the approach to the asymptotic limit. In order to measure any possible discrepancy, parti- cularly in estimates of critical parameters, we have determined empirical limits to the bias and variance error for each parameter. The crucial $\delta$ estimation, in particular, was found to be well behaved.

In the course of preliminary testing we also analyzed a sample of 350 flares, comprising that portion of the sample of 435 "events" used by Wilcox and Schatten (1967) which had precise "time of flare" data. The maximum-likelihood method gave period estimates in reasonable accord with their results. In particular, the test confirms that clustering at a period of 28.85 days is more significant than subsidiary clustering at the period of 27.35 days, but the strength of the test is demonstrated by the fact that the former period is found to be twenty times more likely than the latter period. 
The $6 \frac{1}{2}$ year sample of solar flares was tested for longitudinal structure corresponding to rotational periods in the range $\frac{1}{2}$ day to 150 days, using the trial function of equation (5). The flare series was found to yield a rich spectrum. However, no significant period has been found in the range $\frac{1}{2}$ day to 3 days except for a weak peak in likelihood at 0.9995 days, which most likely corresponds to the universal-time variation in flare reports (Sawyer-Warwick, 1962). Furthermore, no significant structure is associated with the synodic period 115.88 days, the orbital period of Mercury. Much structure was found in the range of periods 13.7 to 31.6 days. The two largest peaks correspond to a period $P_{1}=28,80$ days with a likelihood of $10^{8.5}$, and a period $P_{2}=31.63$ days with a likelihood of $10^{8.3}$. Further data for these peaks are given in Table 1. From the values of the depth of modulation and the standard deviation, the probabilities that these peaks might be produced by a series which is uniformly random are estimated to be $10^{-8.5}$ and $10^{-9.9}$ respectively. The next question to be answered is whether the two periods, $P_{1}$ and $P_{2}$, identified in the above test, might characterize genuine rigid-body rotation in the sun. A test of this hypothesis was to divide the flare series into four sets and to examine the sets separately. It was found that modulation of order $25 \%$ was found at 31.00 days, 30.33 days, 31.51 days and 30.05 days, but periods with values close to $\mathrm{P}_{1}$ didnot always occur. Furthermore, it was found that, when the northern hemisphere and southern hemisphere were considered separately, the period $P_{1}$ showed up for north flares but not for south flares; a peak near $P_{2}$ showed up for both north flares and south flares.

Fearing that the above negative results might be due to the small sample size (the subsamples ranged in number from 120 to 400 ), we proceeded 
to analyze a new sample of 1000 flares (between 1 July 1955 and 31 December 1958) of importance 1+. Modulation of order $25 \%$ was found at three synodic rotation periods: 18.25 days, 21.94 days and 30.90 days. While 30.90 days is in the neighborhood of $\mathrm{P}_{2}$, a rotation period near $p_{1}$ did not occur.

Subsequently, a data sample of about 3000 fiares of importance $1+$ for the period 1955-1961 was subdivided into intervals of about 19 months, and each subsample (with a size of about 700 flares)was analyzed in the above manner. Although strong peaks in the frequency spectrum were found, the peaks were found to differ among sub-samples. It therefore appears that there is no peak or pair of peaks which occur consistently and therefore deserve special significance. Nevertheless, it seemed important to try to understand the origin of the periodicity which shows up in any sample, and for this reason the tests to be described in the next section were carried out.

5. Spectrum of a Reduced Flare Series

Solar-geophysical Data published a compilation of flares which listed the number of the plage in which each flare occurred. For the time interval 1 January 1956 - 31 December 1968, we prepared a "reduced" flare series for which not more than one flare is associated with each plage region. For the three-year interval specified, the reduced series comprises 2,670 flares. Fourier analysis and maximumlikelihood analysis of this sample indicates that the only significant rotation period is about 10 years, corresponding to the sunspot cycle. In order to proceed further, the total sample was divided into six subsamples, by subdividing the time interval into three parts and 
considering the northern and southern hemispheres separately. The results of the maximum-likelihood test of these subsamples are shown in Table 2. In view of the sample size, the maximum values of the likelihood are not considered to be significant. The data suggest that there is a weak periodicity consistent in both northern and southern hemispheres, with a period of between 400 and 500 days for the years 1956 through 1958, and a period of about 370 days for the interval 1959 through 1964.

It was clear that the data of Table 2 do not support the hypothesis that rigid-body rotation affects flare events. We were however aware of the suggestion, made at various times by various authors (Bigg, 1967; Bigg and Mulha11, 1967; Jose, 1965; Kopecky, Mayer and Borovickova, 1952; Trellis, 1966; Wood and Wood, 1965), that solar activity is influenced by planetary configurations. It seemed that this effect, if it exists, might result in weak periodicity, the apparent period drifting in time as the configuration of the major planets changes. It seemed desirable to test this hypothesis.

If the planets affect solar activity, they should have the same influence on both northern and southern hemispheres. Since the planetary configuration changes in a complex way over a 13 year interval, it seemed desirable to formulate a test which does not depend upon spectrum analysis. We decided to test for correlation between the time series for north flares and the time series for south flares.

In carrying out this test, it was necessary to filter out the solar cycle variation which would obviously give a strong north-south correlation which, for present purposes, would be spurious. This filtering was performed as follows:

For any time $t$, we may estimate the average rate of flare occurrences, averaging over a time interval $T$. This rate will be written as 
$R_{N}(t ; T), R_{S}(t ; T)$, for the northern and southern hemispheres respectively. Formation of these averages (equivalent to forming "running means") effectively filters out structure with a period less than $T$.

For the purpose specified, it was desirable to eliminate structure with a period less than about 20 days. It was also desirable to filter out structure with a period appreciably greater than 400 days, so as to avoid contamination by the solar cycle. We therefore formed two averaged rates, adopting the periods $\mathrm{T}_{1}=20$ days, $\mathrm{T}_{2}=400$ days. The step interval $t_{i+1}-t_{i}$, was ten days. The series defined by

$$
\left.\begin{array}{l}
X_{N}\left(t_{i}\right)=R_{N}\left(t_{i} ; T_{1}\right)=R_{N}\left(t_{i} ; T_{2}\right), \\
x_{S}\left(t_{i}\right)=R_{S}\left(t_{i} ; T_{1}\right)-R_{S}\left(t_{i} ; T_{2}\right),
\end{array}\right\}
$$

represent time-series for north flares and south flares from which structure of period shorter than $T_{1}$ or longer than $T_{2}$ has been eliminated. We next evaluated the correlation coefficient $\xi$ for these two filtered time series:

$$
\xi=\frac{\sum_{i} x_{N}\left(t_{i}\right) x_{S}\left(t_{i}\right)}{\left\{\sum_{j}\left(x_{N}\left(t_{j}\right)\right)^{2}\right\}^{\frac{1}{2}}\left\{\sum_{j}\left(x_{S}\left(t_{j}\right)\right)^{2}\right\}^{\frac{1}{2}}} .
$$

It was found that, for the reduced $f l$ are series discussed in this section, $\xi=.051$. With a 13-year interval and 10-day "bin" size, the number of bins $N_{B}=475$. For independent random time series $x_{N}\left(t_{i}\right), x_{S}\left(t_{i}\right)$, the square-root of the expectation value of $\xi^{2}$ is $N_{B}^{-\frac{1}{2}}$, or .046. This test therefore shows that there is no significant correlation between north flares and south flares and, in consequence, 
no reason to believe that flares are affected by a mechanism such as solar rotation or planetary gravitational fields.

6. Discussion

The results of this study may be stated as follows:

1) In searching for periodic modulation of flare (or sunspot) activity, it is essential to work with a "reduced" time series, for which only one event is assigned to any one active region. If this is not done, the time series will have a rich spectrum originating in recurrent activity in long-lived centers of activity.

2) Spectrum analysis of long samples of reduced solar-flare time series shows no structure which can be identified as statistically significant.

3) A test for correlation between reduced flare series in the northern and southern hemispheres shows no evidence forsuch a correlation. This result lends no support to the hypothesis that solar activity is modulated by planetary influences.

Although the results of the tests reported in this article have been negative, it must be emphasized that this does not prove that there is no genuine periodic modulation of solar activity, or that there are no planetary influences. Recent work by Wilcox, Schatten, Tannenbaum and Howard (1970) indicates that weak-field structure exhibits rigidrotation modulation, and it is possible that investigation of even larger flare samples would show that a very weak modulation of this type exists in flare activity. Similarly, it is possible that planetary modulation (if any) is so weak that it does not show up in our tests, 
but does show up in a more sensitive and specific test, such as that carried out by Bigg and Mulhall (1967).

Nevertheless, we may safely conclude that, if rigid-body modulation or planetary modulation does exist, it is of such a low amplitude as to be useless to anyone concerned with the prediction of solar activity.

\author{
Acknowledgements \\ We are indebted to $\mathrm{Mr}$. Peter Coleman for assistance in the data \\ processing. This work was supported by the Air Force office of Scientific \\ Research under Contract F44620-69-C-0008 and by the National Aeronautics \\ and Space Administration Grant NGL 05-020-272. In addition, one of us \\ (G.V.H.) would like to acknowledge support for part of this work from \\ the Graduate Council of the University of California at Irvine.
}




\section{References}

Bartels, J.: 1932, Terrestrial Magnetism and Atmospheric Electricity, $37: 1$ 。

Bigg, E.K.: 1967, Astron, J。 72, 463 .

Bigg, E.K, and Mulhall, P.S.: 1967, Proc, Astron, Soc, Aust, I, 53.

Carrington, R.C.: 1863, Observation of the Spots on the Sun from November 9, 1853, to March 24, 1861, Williams and Norgate, London and Edinburgh.

Cox, D.R. and Lewis, P.A.W.: 1966, The Statistical Analysis of a Series of Events, Methuen, London.

Dickie, R.H. and Goldenberg, H.M.: 1967, Phys. Rev. Letters, 18, 313.

Explanatory Supplement to the Astronomical Ephemeris and the American Ephemeris and Nautical Almanac: 1961, H.M. Stationery Office, London.

Guss, D.E.: 1964, Phys. Rev. Letters, 13, 363.

Jenkins, G.M.: 1967, Time Series Analysis Papers (ed. E. Parzen), Holden-Day, San Francisco, p. 98.

Jose, P.D.: 1965, Astron. J., 70, 193.

Kopecky, M., Mayer, P. and Borovickova, V.: 1952, Bull Cent, Astron. Inst. Czech, $\underline{3}_{2} 37$.

Losh, H.M.: 1938, Pub. Obs. Univ. Michigan, ㄱ, No. 5, 127.

Newton, H.W. and Nunn, M.L.: 1951, Mon. Not, Roy, Astron. Soc, , 111, 413.

Rao, C.R.: 1965, Linear Statistical Inference and Its Applications, J. Wiley and Sons, New York, Ch, 5 .

Sawyer-Warwick, C.S.: 1962, 1964, 1966, National Bureau of Standards Lists of IGY Flares, with Normalized Values of Importance and Area, I.G.Y. Solar Activity Report Series Nos, 17, 29 and 33, High Altitude Observatory, Boulder, Colorado.

Saw yer-Warwick, C.S.: 1965, Astrophys, J., 141, 500.

Trellis, M.: 1966, C.R.Acad。Sci, Paris, 262, 376.

Vitinskii, Yu I。: 1960, Izv. Glav。Obs。Pulkova, 21, 96.

Wilcox, J.M. and Ness, N.F.: 1965, J。Geophys。Res., 70, 5793 。

Wilcox, J.M. and Schatten, K.H.: 1967, Astrophys。 J, 147, 364 .

Wilcox, J.M., Schatten, K.H., Tannenbaum, A.S. and Howard, R。: 1970, submitted to Sol. Phys.

Wood, R.M. and Wood, K.D.: 1965, Nature, 208, 129 . 


\section{TABLE 1}

Principal Components of Spectrum of 1119 Flares

Occurring Between 1 July 1955 and 31 December 1961.

\begin{tabular}{lccccccc}
\hline & & & & & & & \\
Synodic Period (days) & 28.80 & \pm & .06 & 31.63 & \pm & .05 \\
Log Relative Likelihood & 8.5 & & & 8.3 & & \\
Depth of Modulation $\delta$ & .254 & \pm & .041 & .269 & \pm & .041 \\
Phase Angle $Y$ (deg.) & 315 & \pm 30 & 236 & \pm 23 \\
\end{tabular}




\section{\begin{tabular}{llllll} 
T A B L E & 2 \\
\hline
\end{tabular}}

Principal Components of Spectrum of 2670 Flares of Reduced

Flare Series Occurring Between 1 January 1956 and 31 December 1968

\begin{tabular}{|c|c|c|c|c|c|c|}
\hline Time Interval ${ }^{*}$ (days) & \multicolumn{2}{|c|}{$0-1203.385$} & \multicolumn{2}{|c|}{$1203.385-3324.06$} & \multicolumn{2}{|c|}{$3324.06-4745.28$} \\
\hline Hemisphere & $\mathrm{N}$ & $\mathbf{s}$ & $\mathbf{N}$ & $\mathbf{S}$ & N & $\mathbf{S}$ \\
\hline Number of Flares & 466 & 434 & 540 & 270 & 605 & 355 \\
\hline Log Likelihood & 3.95 & 2.38 & 2.91 & 1.47 & & \\
\hline Synodic Period (days) & $476.5 \pm 23.8$ & $417.5 \pm 22.8$ & $380.0 \pm 10.9$ & $360.0 \pm 12.4$ & sign & $\operatorname{can} t$ \\
\hline Depth of Modulation & $0.27 \pm 0.06$ & $0.22 \pm 0.07$ & $0.22 \pm 0.06$ & $0.22 \pm 0.08$ & & \\
\hline Phase Angle (deg) & $86.6 \pm 34.0$ & $129.1 \pm 42.6$ & $151.0 \pm 70.0$ & $12.0 \pm 90.0$ & & \\
\hline
\end{tabular}

* Interval measured from 00.001, 1 January 1956. 


\section{DOCUMENT CONTROL DATA - R \& D}

(Security classification of title, body of abstract and indexing annotation must be entered when the overall report is classified) 1. ORIGINATING ACTIVITY CORPORGIO Institute for Plasma Research Stanford University

Stanford, California 94305

3. REPORT TITLE

\section{LONGITUDE DISTRIBUTION OF SOLAR FLARES}

4. DESCRIPTIVE NOTES (Type of report and inclusive dates)

Scientific Interim

5. AU THOR(S) (First name, middle initial, last name)

P.C.W.Fung, P.A. Sturrock, P. Swtizer, G, Van Hoven

\begin{tabular}{|c|c|}
\hline $\begin{array}{l}\text { 6. REPORT DATE } \\
6 \text { NOVEmber } 1970\end{array}$ & \begin{tabular}{|c|c|} 
7a. TOTAL NO. OF PAGES & 7b. NO. OF REFS \\
19 & 22
\end{tabular} \\
\hline $\begin{array}{l}\text { 8a. FONTRACT OR GRANT NO. } \\
\text { F44620-69-C-0008 } \\
\text { b. PROJECT NO. 9751-O1 }\end{array}$ & $\begin{array}{l}\text { 9a. ORIGINATOR'S REPORT NUMGER(S) } \\
\text { SU-IPR Report No. } 393\end{array}$ \\
\hline $\begin{array}{l}\text { c. } 61102 \mathrm{~F} \\
\text { d. } 681302\end{array}$ & $\begin{array}{l}\text { 9b. OTHER REPORT NO(S) (Any other numbers that may be assigned } \\
\text { this report) }\end{array}$ \\
\hline
\end{tabular}

This document has been approved for public release and sale; its distribution is unlimited.

11. SUPPLEMENTARY NOTES 2. SPONSORING MILITARY ACTIVITY

AFOSR-SRPN

TECH, OTHER 1400 Wilson Blvd. Arlington, Virginia 22209

Statistical tests, based on the maximum-likelihood method, have been performed on flare series extending over several years. If all flares in each plage region are taken into account, a rich spectrum is obtained. If one carries out similar analysis of a "reduced" flare series, which includes at most one flare from each plage region, the spectrum is almost devoid of structure, and what structure does remain is not statistically significant. The inference is that solar activity does not display genuine rigid-rotation modulation, but that repeated events from individual centers of activity yield modulation which may be mistaken for rigid-rotation modulation.

A test for correlation between reduced flare series for the northern and southern hemispheres gives no significant correlation. This test therefore yields no support to the hypothesis that solar activity is modulated by planetary effects. 


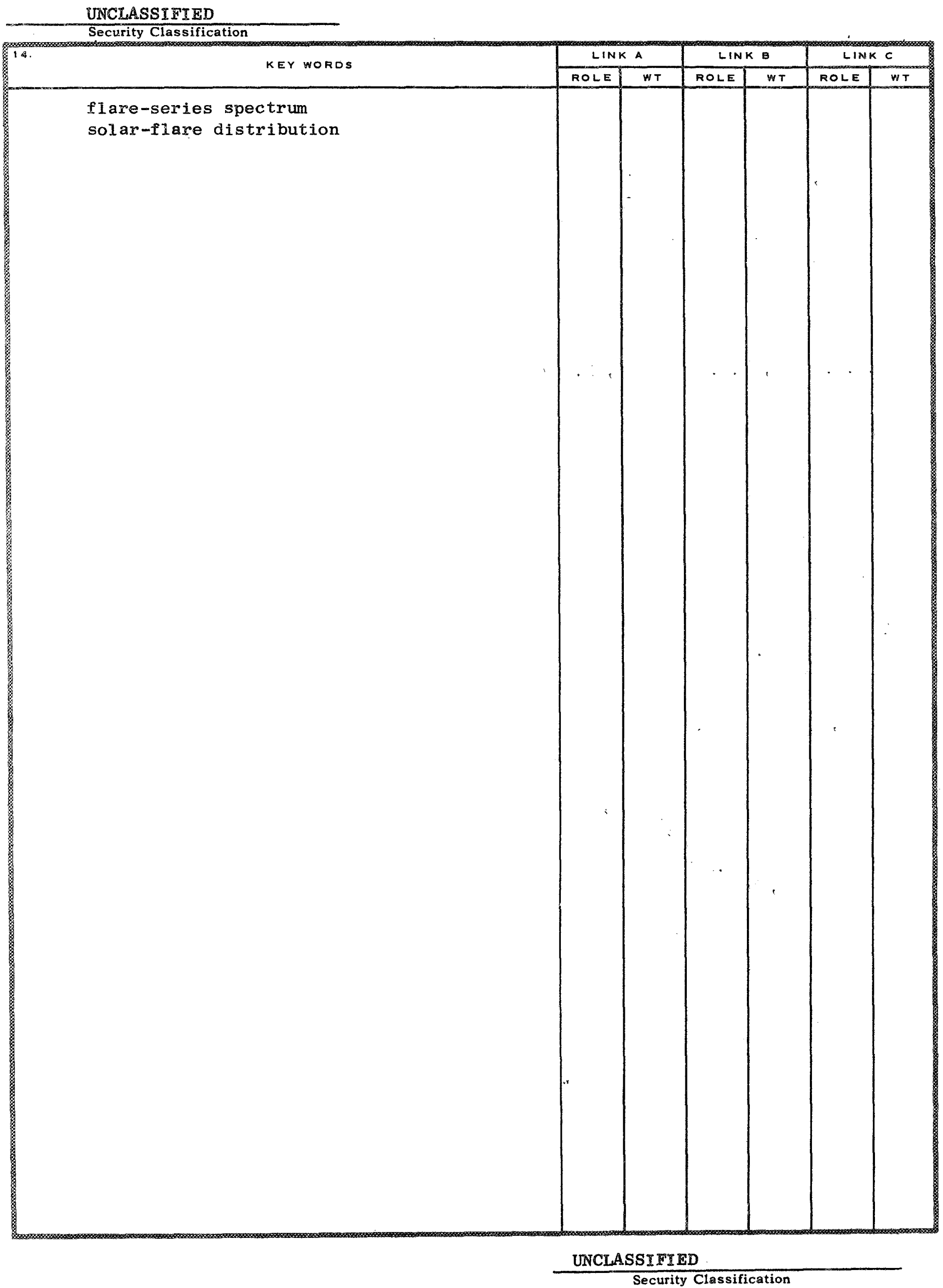

